\title{
Use of a water treatment sludge in a sewage sludge dewatering process
}

\author{
Justyna Górka ${ }^{1,}$, Małgorzata Cimochowicz-Rybicka ${ }^{1}$, and Małgorzata Kryłów ${ }^{1}$ \\ ${ }^{1}$ University of Technology, Department of Environmental Engineering, 24 Warszawska, Cracow 31- \\ 155 , Poland
}

\begin{abstract}
The objective of the research study was to determine whether a sewage sludge conditioning had any impact on sludge dewaterability. As a conditioning agent a water treatment sludge was used, which was mixed with a sewage sludge before a digestion process. The capillary suction time (CST) and the specific filtration resistance (SRF) were the measures used to determine the effects of a water sludge addition on a dewatering process. Based on the CST curves the water sludge dose of $0.3 \mathrm{~g}$ total volatile solids (TVS) per $1.0 \mathrm{~g}$ TVS of a sewage sludge was selected. Once the water treatment sludge dose was accepted, disintegration of the water treatment sludge was performed and its dewaterability was determined. The studies have shown that sludge dewaterability was much better after its conditioning with a water sludge as well as after disintegration and conditioning, if comparing to sludge with no conditioning. Nevertheless, these findings are of preliminary nature and future studies will be needed to investigate this topic.
\end{abstract}

\section{Introduction}

Dewatering of sludge is a very important stage of the sludge processing. It reduces both sludge mass and volume, which consequently reduces costs of a further sludge processing, facilitates its transport and allows for its thermal processing. Generally, a sewage sludge has a very high water content (92-99.5\%), which contributes to high costs of its further processing. So even a slight decrease of a water content may result in a significant reduction of a sludge volume, facilitating its subsequent disposal [1]. It is also worth noting that a growing acceptance of a sludge incineration, as the final sewage sludge disposal, stimulates a search for methods that would intensify dewatering processes.

At sewage treatment plants, it is mostly mixed sludge that is dewatered after thickening and anaerobic or aerobic digestion. Processing of a sewage sludge is difficult because the sludge is rich in organic compounds that form gel-like webs and may slow down dewatering [2]. Therefore, different methods of sludge conditioning are applied during its processing and dewatering. Sludge conditioning modifies a structure and properties of the processed sludge and enables a more efficient removal of water captured within sludge flocks [3]. Nevertheless, the selection of optimal and most efficient measures used in this process remains still an open question [4]. Sludge conditioning can be carried out with physical or chemical methods.

Physical methods of sludge conditioning include:

*Corresponding author: justynagrka@gmail.com 
- High temperature,

- Freezing,

- Addition of conditioners that modify a sludge structure, such as: coal dust, cement dust and ash.

Chemical conditioning, on the other hand, involves supplementing sludge with some organic (polymerized cationic, anionic or neutral polyelectrolytes) or inorganic (iron and aluminum salts) compounds. In addition to the commonly used sludge conditioning techniques, also sludge from water treatment processes may be used as such supplementing agent that facilitates a dewatering process; it may contain aluminum or iron precipitates, depending on the water treatment methods used.

Currently, there is a number of studies underway focusing on this topic and investigating the issues on management and utilization of a water treatment sludge [5-7] . The authors have shown that conditioning of a sewage sludge with a water sludge improves its dewaterablity. The addition of sludge from water treatment plants, especially the ones employing coagulation with aluminum salts, improved dewaterablity of excess sludge. Moreover, sludge filtration properties improved along with a larger fraction of a water treatment sludge added to a sewage sludge [8]. Dewaterablity of digested sludge mixed with aluminium precipitates was also investigated and it was confirmed that they improved the sewage sludge dewaterblility [9].

The objective of the research was to determine whether sewage sludge conditioning had any impact on sludge dewaterability. As a conditioning agent a water treatment sludge was used, which was mixed with the digested sludge before another digestion. Such an interference in anaerobic digestion (modification of sludge before the process) affects the sludge final dewaterability. In the next step, the authors tried to assess how disintegration of a water sludge affects sewage sludge dewaterablity. The findings were of preliminary nature and future studies would be needed to thoroughly investigate this topic.

\section{Material and methods}

A sewage sludge (SS) from a municipal wastewater treatment plant with a capacity of $150000 \mathrm{~m}^{3} \cdot \mathrm{d}^{-1}$ was used for the study. The sludge was a mixture of primary and excess sludge. The water treatment sludge (WTS) came from a municipal water treatment plant with a capacity of $100000 \mathrm{~m}^{3} \cdot \mathrm{d}^{-1}$. The sludge was produced during coagulation of water with aluminum coagulant (PAX) and a sand filtration. In order to compare the samples for filtration properties tests were used:

- Sewage sludge from wastewater treatment plant mixed with water treatment sludge from water treatment plant,

- Sewage sludge from wastewater treatment plant mixed with water treatment sludge from water treatment plant - the mixture was digested.

The research included respirometric batch tests, which had been employed for energy research; enabled to analyze both quantity and quality of a fermentation gas. The digestion process was carried out under mesophilic conditions at $35{ }^{\circ} \mathrm{C}$ for 21 days in three separate runs. The sludge samples with of 1 liter volume had concentrations of 4.0-5.0 g TVS $\cdot \mathrm{dm}^{-3}$. To ensure the appropriate process conditions, $\mathrm{pH}$ of the samples was adjusted to $\mathrm{pH}=7.0$ with $\mathrm{NaOH}$. The sample was purged with technical nitrogen for three minutes, before measurements were taken. The analysis during anaerobic batch tests also included: dry solids (TS), total volatile solids (TVS), chemical oxygen demand (COD), alkalinity, pH, ammonia nitrogen and total phosphorus, measured according to the current EU standards.

In the first stage of the study, the optimum dose of a WTS was selected; a selection was done while testing different proportions of a water treatment sludge to a sewage sludge 0.1-1.0 g TVS of a water treatment sludge per $1.0 \mathrm{~g}$ TVS of a sewage sludge. The criterion 
for selecting the optimum water treatment sludge dose included both an improvement in sludge dewaterability and a biogas production (higher efficiency of the digestion process not included in the article).

In the next stage of the study, to further improve sludge dewaterability as well as the digestion efficiency, the water treatment sludge was disintegrated and mixed with sewage sludge. Disintegration was carried out at the most favorable disintegration parameters. According to the previous research (not included in the article), it was found that the optimum disintegration parameters were:

- Ultrasonic disintegration: intensity $24 \mathrm{~kW} \cdot \mathrm{m}^{-2}$, time $10 \mathrm{~min}$,

- $\quad$ Thermal disintegration: temperature $55^{\circ} \mathrm{C}$, time $60 \mathrm{~min}$.

The tests series were run on the following materials:

- $\mathrm{SS}$,

- $\quad$ SS+WTS in a ratio of 1:0.3,

- $\quad$ SS+WTS (WTS after thermal disintegration) in a ratio of 1:0.3,

- $\quad$ SS+WTS (WTS after ultrasonic disintegration) in a ratio of 1:0.3.

Sludge dewaterability was measured by a capillary suction time (CST) and a specific filtration resistance (SRF). The capillary suction time measurement was based on the Baskerville and Galle standard PN-EN 1407-1: 2007, which measures the passage of the head filtrate layer due to a suction force of the Whatman filter paper.

The specific filtration resistance was determined based on the PN-EN 14701-2 standard. This method follows the flow of a fluid through a porous medium in accordance with the Darcy's law. The specific resistance to filtration is determined by pouring a suitable volume of sludge into a filtering device, allowing the liquid to be filtered under constant vacuum or pressure $(50 \mathrm{kPa})$, while recording the amount of filtrate over time. The specific resistance to filtration is calculated as follows:

$$
S R F=\frac{2 \cdot \Delta p \cdot A^{2} \cdot b}{\mu \times m}\left[m \cdot k^{-1}\right]
$$

where:

$\Delta p$ - pressure drop across the filter,

$A$ - filtration area,

$b$ - the slope of the linear part of the curve obtained by plotting $t / V$ vs. V (Fig. 1),

$\boldsymbol{\mu}$ - the viscosity of filtrate at the temperaturę of the sludge,

$m$ - the mass of solids deposited on the filtering medium per unit volume of filtrate.

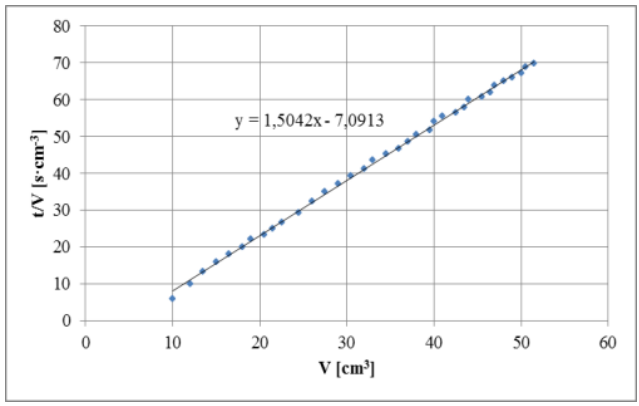

Fig. 1. Linear part of the curve obtained by plotting $\mathrm{t} / \mathrm{V}$ vs. V. 
Based on the $f(V)=t / V$ graph (Fig. 1) the water content can be estimated in the individual phases of sludge filtering and the water volume in the sludge according to the method of attachment to a solid structure.

\section{Results}

The physic-chemical characteristics of the WTS and the SS prior to the anaerobic digestion are shown in Table 1 . The water treatment sludge had lower values of such parameters as; TVS (by about 48\%), COD (by 66\%), phosphorus (by 70\%), total nitrogen (by $73 \%$ ) and ammonium nitrogen (by $88 \%$ ) than the sewage sludge.

Table 1. The physic-chemical characteristics of the sludge.

\begin{tabular}{llcc}
\hline Parameter & & Water treatment sludge & Sewage sludge \\
\hline $\mathrm{pH}$ & {$[-]$} & 6.65 & 7.02 \\
$\mathrm{TS}$ & {$\left[\mathrm{gTS} \cdot \mathrm{dm}^{-3}\right]$} & 35.13 & 32.17 \\
$\mathrm{TVS}$ & {$\left[\mathrm{gTVS} \cdot \mathrm{dm}^{-3}\right]$} & 9.87 & 19.11 \\
$\mathrm{COD}$ & {$\left[\mathrm{mgO}_{2} \cdot \mathrm{dm}^{-3}\right]$} & 8000 & 24000 \\
$\mathrm{sCOD}$ & {$\left[\mathrm{mgO}_{2} \cdot \mathrm{dm}^{-3}\right]$} & 74 & 320 \\
$\mathrm{TP}$ & {$\left[\mathrm{mgP} \cdot \mathrm{dm}^{-3}\right]$} & 174 & 581 \\
$\mathrm{TN}$ & {$\left[\mathrm{mgN} \cdot \mathrm{dm}^{-3}\right]$} & 448 & 1646 \\
$\mathrm{~N}_{\mathrm{NH} 4}$ & {$\left[\mathrm{mgN}_{\mathrm{NH} 4} \cdot \mathrm{dm}^{-3}\right]$} & 78 & 638 \\
\hline
\end{tabular}

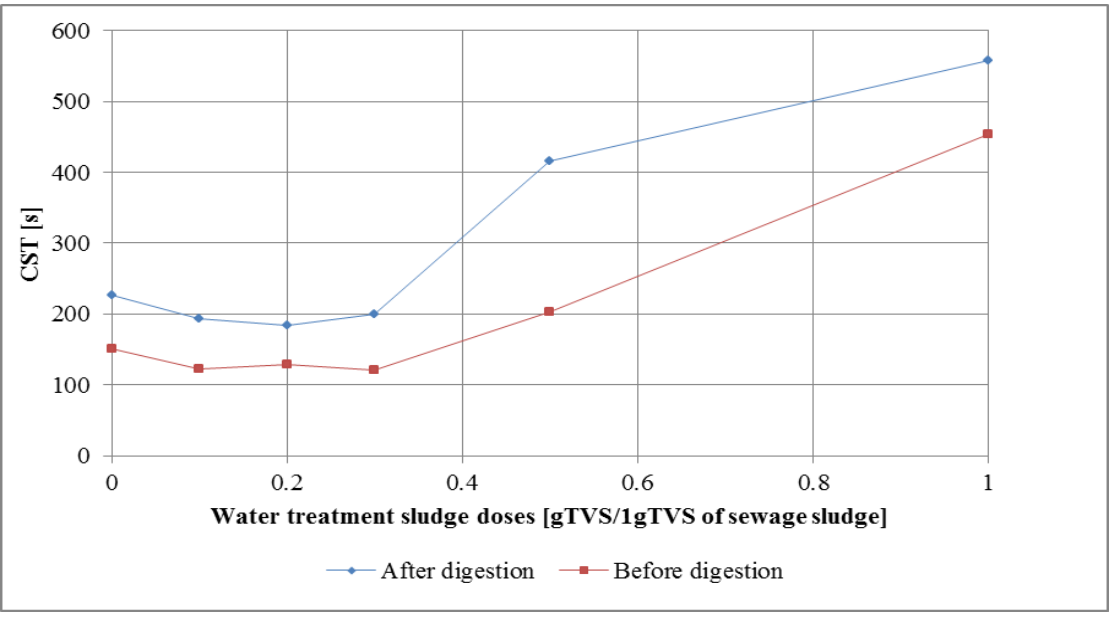

Fig. 2. CST of the tested sludge before and after digestion.

In order to determine the optimum ratio of a water tratment sludge to a sewage sludge, the changes of the CST were investigated for the tested sludge, before and after the digestion process. The tests were run for different doses of the water treatment sludge and their results are shown in Figure 2. It is worth noting that the CST values observed before the digestion process were lower than the ones after digestion. The average CST values for sludge that had not been conditioned with water treatment sludge and was not digested were $150 \mathrm{~s}$, and they were $66 \%$ lower than the ones after digestion. It has also been observed that above the dose of $0.3 \mathrm{~g}$ TVS of water treatment sludge/g TVS of sewage sludge the CST values increased, which indicates that the use of a water treatment sludge as 
a sewage sludge conditioner may adversely affect sludge dewaterability. The optimal ratio of water to sewage sludge proved to be 1:0.3 (1.0g TVS of sewage sludge to $0.3 \mathrm{~g}$ TVS of water treatment sludge). The average capillary suction time for the sample (before digestion) was $120 \mathrm{~s}$ and it was $60 \%$ lower than the ones observed for digested samples. The optimal ratio of 1:0.3 was selected for the further studies.

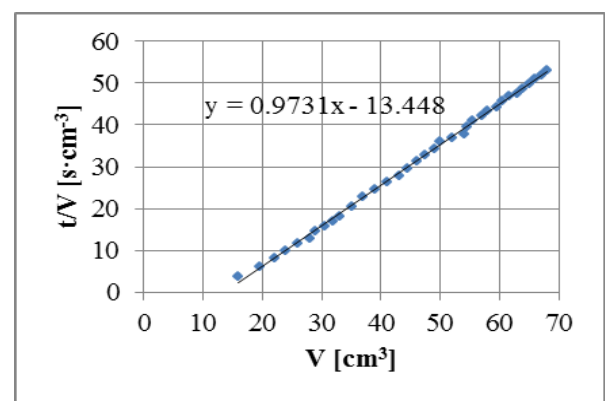

a)

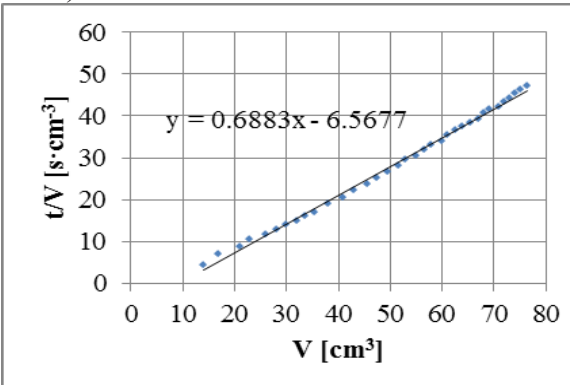

c)

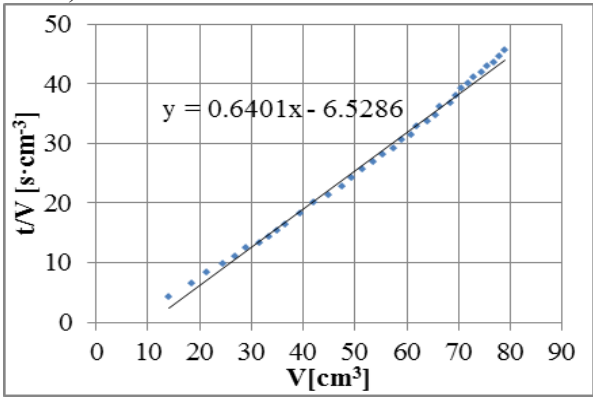

e)

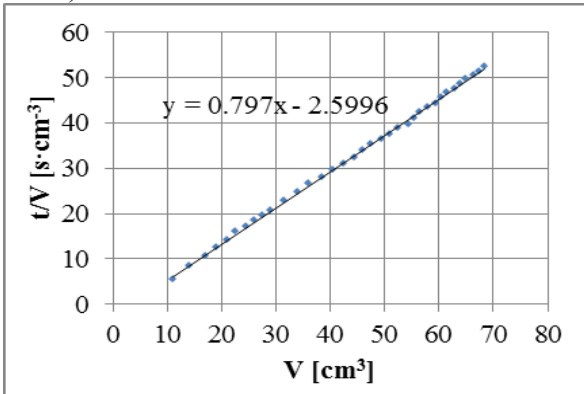

g)

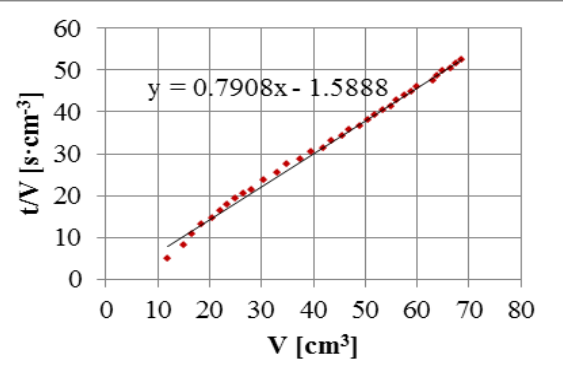

b)

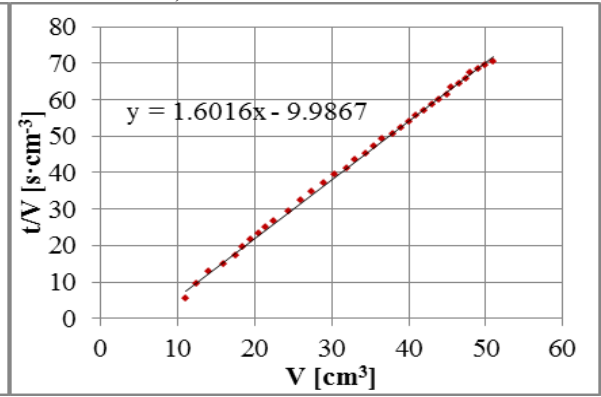

d)

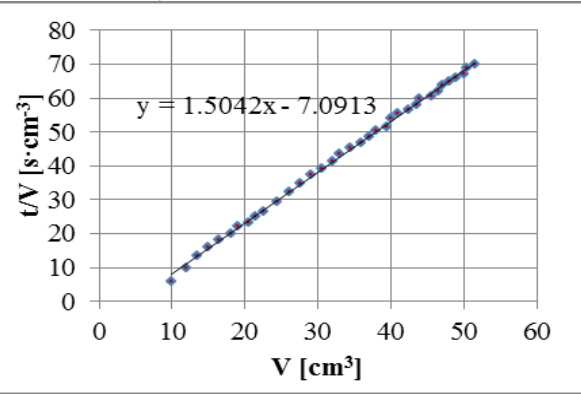

f)

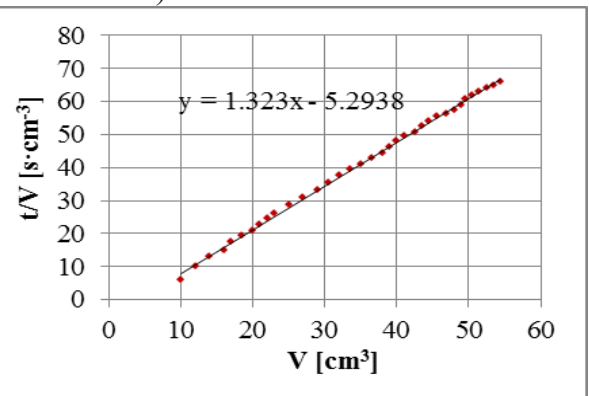

h)

Fig. 3. Filtration curves: $\mathrm{a}-\mathrm{SS}, \mathrm{b}-\mathrm{SS}$ after digestion, $\mathrm{c}-\mathrm{SS}+\mathrm{WTS}(1: 0.3), \mathrm{d}-\mathrm{SS}+\mathrm{WTS}(1: 0.3)$ after digestion, e - SS+WTS thermal (WTS after thermal disintegration) in a ratio of 1:0.3, $\mathrm{f}-$ 
SS+WTS thermal (WTS after thermal disintegration) in a ratio of 1:0.3 after digestion, $g-$ SS+WTS ultrasonic (WTS after ultrasonic disintegration) in a ratio of 1:0.3, $\mathrm{h}-\mathrm{SS}+$ WTS ultrasonic (WTS after ultrasonic disintegration) in a ratio of 1:0.3 after digestion.

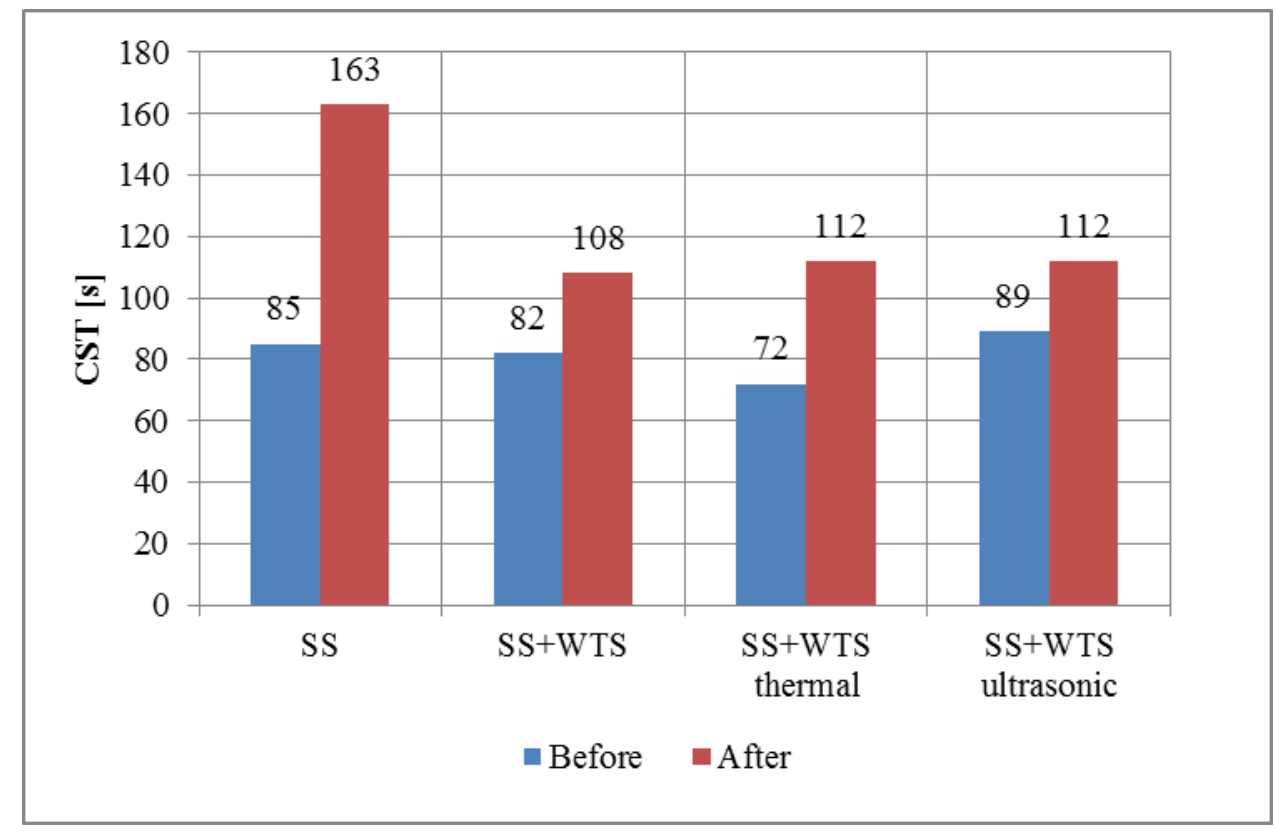

Fig. 4. CST for the sludge samples before and after digestion.

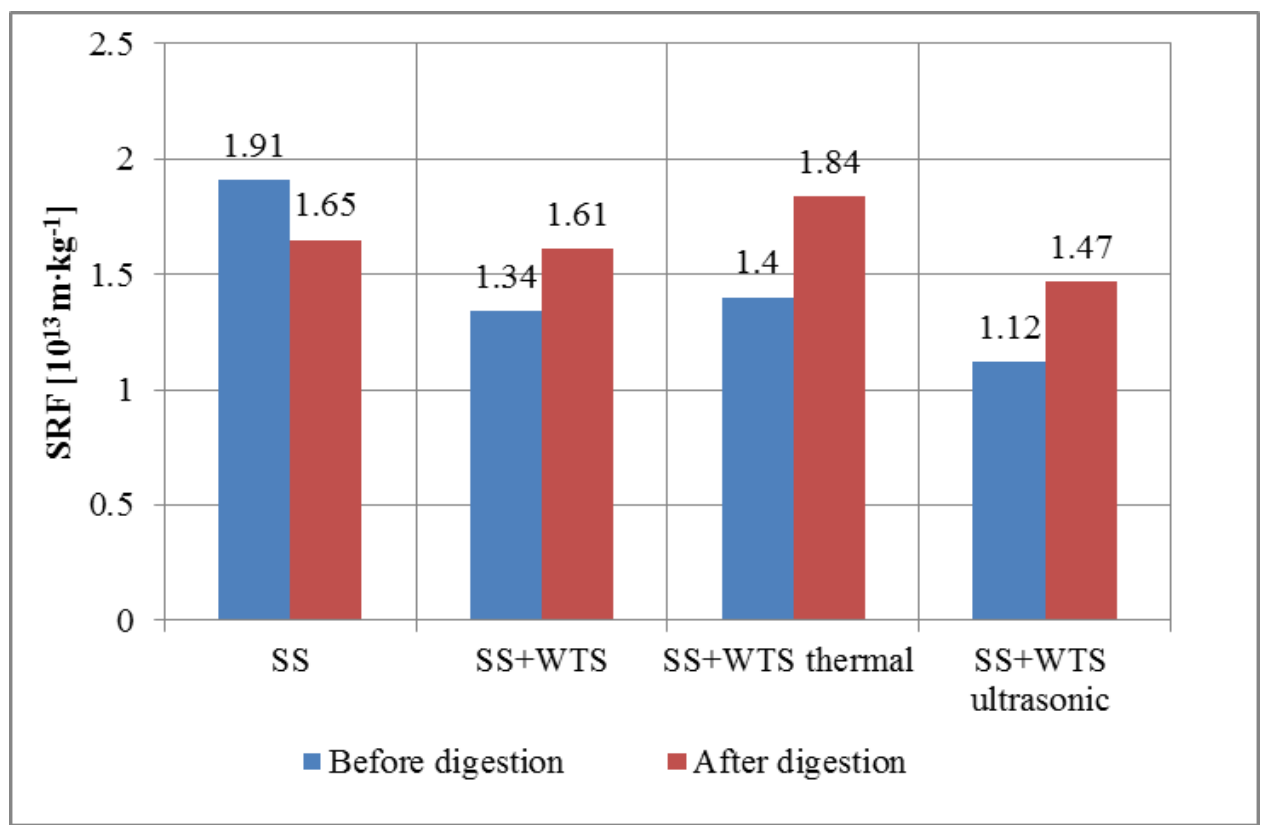

Fig. 5. SRF of samples before and after digestion.

Before sludge digestion, a slight difference in capillary suction times (a change from 72 to 89 s) was observed (Fig. 4). This indicates that both conditioning with a water treatment sludge and using disintegration methods hardly effect sewage sludge filtration 
properties. In turn, an anaerobic digestion resulted in a major increase of the CST in all samples - ranging from 108 to $163 \mathrm{~s}$. The highest CST values were observed in the sewage sludge (163 s). The sewage samples conditioned with a water treatment sludge (regardless of disintegration) had the CST about $66 \%$ lower than the sewage sludge samples. A thermal disintegration, like an ultrasonic one, did not significantly affect a sludge dewatering ability.

Analizing the Figure 3, it can be concluded that with increasing dry solids in the sludge cake, the SRF increased. Dewatering of sewage sludge in a vacuum filter, during the SRF measurement, increased filtration resistance (Figure 5) for samples conditioned with water treatment sludge after anaerobic digestion: for sludge conditioned with a water treatment sludge by $17 \%$ and for sludge conditioned with a water treatment sludge after ultrasonic and thermal degradation by $24 \%$. In turn, SRF for only sewage sludge was lower by $15 \%$.

\section{Conclusions}

Based on the research, the following conclusions were made:

1. Conditioning of a sewage sludge with sludge from water treatment processes improved a sewage sludge dewatering characteristic. The studies have shown that conditioning of sludge with sludge from water treatment processes after digestion resulted in higher sludge dewaterability i.e. lower CST values.

2. The changes of the CST values depended on the dose of a water treatment sludge; the optimum dose of a water treatment sludge was $0.3 \mathrm{~g}$ TVS per $1 \mathrm{~g}$ TVS of sewage sludge.

3. The study clearly stated that anaerobic digestion increased the CST and SRF values. However, for sludge conditioned with a water treatment sludge as well as for a disintegrated water sludge, the CST values were lower than for a sewage sludge.

4. Both thermal and ultrasonic disintegration of sludge from water treatment processes did not significantly affect sewage sludge dewaterability.

\section{References}

1. M. Christensen, K. Keiding, P.H. Nielsen, M.K. Jorgensen. Wat. Res., 82 (2015).

2. S. Skinner, L. Studer, D. Dixon, P. Hillis, C. Rees, R. Wall, R. Cavalida, S. Usher, A. Stickland, P. Scales. Wat. Res., 82 (2015).

3. P. Wolski, L. Wolny. Rocz. Ochr. Środ., 13 (2011).

4. M. Dębowski, M. Zieliński, M. Krzemieniewski. Ochr. Środ., 30 (2008).

5. T. Ahmad, K. Ahmad, M. Alam. Journ. Clean. Prod., 124 (2016).

6. K. Dassanayake, G. Jayasinghe, A. Surapaneni, C. Hetherington. Was. Man., 38 (2015).

7. M. Cimochowicz-Rybicka, J. Górka. Przem. Chem., 96/8 (2017).

8. J. Lai, J. Liu. Wat. Sc. \& Tech., 9 (2004).

9. Y. Yang, Y. Zhao, A. Babatunde, P. Kearney. Wat. Envir., 13 (2007). 